\title{
Prognostic value of tumor infiltrating lymphocytes combined with PD-L1 expression for patients with solitary colorectal cancer liver metastasis
}

\author{
Binyi Xiao $^{1 \# \wedge}$, Jianhong Peng ${ }^{1 \#}$, Yongchun Wang ${ }^{2 \#}$, Yuxiang Deng ${ }^{1}$, Qingjian $\mathrm{Ou}^{1,2}$, Xiaojun $\mathrm{Wu}^{1}$, \\ Junzhong Lin ${ }^{1}$, Zhizhong Pan $^{1}$, Lin Zhang ${ }^{3,4}$ \\ ${ }^{1}$ Department of Colorectal Surgery, Sun Yat-sen University Cancer Center, State Key Laboratory of Oncology in South China, Collaborative \\ Innovation Center for Cancer Medicine Guangzhou, Guangzhou, China; ${ }^{2}$ Department of Experimental Research, Sun Yat-sen University Cancer \\ Center, State Key Laboratory of Oncology in South China, Collaborative Innovation Center for Cancer Medicine Guangzhou, Guangzhou, China; \\ ${ }^{3}$ Department of Medicine Laboratory, Sun Yat-sen University Cancer Center, State Key Laboratory of Oncology in South China, Collaborative \\ Innovation Center for Cancer Medicine Guangzhou, China; ${ }^{4}$ Department of Clinical Laboratory, Yunfu People's Hospital, Yunfu, China \\ Contributions: (I) Conception and design: Z Pan, L Zhang; (II) Administrative support: Z Pan, L Zhang; (III) Provision of study materials or patients: \\ Y Wang, Y Deng, Q Ou, X Wu, J Lin; (IV) Collection and assembly of data: B Xiao, J Peng; (V) Data analysis and interpretation: B Xiao, J Peng; (VI) \\ Manuscript writing: All authors; (VII) Final approval of manuscript: All authors. \\ \#These authors contributed equally to this work. \\ Correspondence to: Zhizhong Pan; Lin Zhang. Sun Yat-sen University Cancer Center, 651 Dongfeng Road East, Guangzhou, China. \\ Email: panzhzh@sysucc.org.cn; zhanglin@sysucc.org.cn.
}

Background: The aim of this study was to assess the prognostic value of CD8+ tumor infiltrating lymphocytes (TIL) combined with programmed cell death-ligand 1 (PD-L1) expression for patients with solitary colorectal cancer liver metastasis (SCLM) undergoing R0 resection

Methods: Patients undergoing curative hepatectomy for SCLM were reviewed. Immunohistochemical multiplex technique was used for quantifying CD8+ TIL, and immunohistochemical staining was used for assessing PD-L1 expression. The tumor immune microenvironment (TIME) was classified as strong for high CD8+ TIL and low PD-L1, weak for low CD8+ TIL and high PD-L1, and mild for the rest. Recurrencefree survival (RFS) and overall survival (OS) was compared between these groups.

Results: Among the 94 patients included, a high CD8+ TIL and high PD-L1 expression was observed in $51(54.3 \%)$ and $47(50.0 \%)$ patients, respectively. Strong, mild, and weak TIME was observed in 24 (25.5\%), $42(44.7 \%)$, and 28 (29.8\%) patients, respectively. Patients with a high CD8+ TIL had a significant longer RFS than patients with a low CD8+ TIL (3-year RFS rate, 71.6\% vs. 55.3\%, $\mathrm{P}=0.018$ ). The 3-year RFS rate in the strong TIME group was significantly higher than that in the mild and weak TIME groups (89.5\% vs. $71.7 \%$ and $28.8 \%, \mathrm{P}<0.001)$, as was the 3 -year rate of $\mathrm{OS}(93.8 \%$ vs. $81.8 \%$ and $61.6 \%, \mathrm{P}<0.001)$. CD $8+$ TIL combined with PD-L1 expression showed better predicting accuracy for RFS than CD8+ TIL alone.

Conclusions: The density of CD8+ TIL combined with PD-L1 expression in liver metastasis was a predictor of RFS for patients with SCLM undergoing R0 resection, and therefore can be used for guiding the postoperative treatment of these patients.

Keywords: Colorectal cancer (CRC) liver metastasis; recurrence-free survival (RFS); tumor infiltrating lymphocytes (TIL); programmed cell death-ligand 1 (PD-L1)

Submitted Mar 23, 2020. Accepted for publication Aug 16, 2020.

doi: $10.21037 / \mathrm{atm}-20-2762 \mathrm{a}$

View this article at: http://dx.doi.org/10.21037/atm-20-2762a

$\wedge$ ORCID: 0000-0002-7675-3207. 


\section{Introduction}

Colorectal cancer (CRC) is one of the leading causes of cancer-related death, with an estimate of 0.81 million mortality globally (1). Liver metastasis is the main reason for treatment failure, occurring in nearly $50 \%$ of CRC patients along the course of their disease (1-3). Surgery is the major potentially curative therapy for patients with CRC liver metastasis (CRCLM), but nearly 70\% experience recurrence even after R0 resection $(4,5)$. The Clinical Risk Score, proposed by Fong et al., is a widely used scoring system for patients with CRCLM (6). Patients with a higher score are more likely to develop new lesions after hepatectomy, and thus are recommended for postoperative chemotherapy (7). Predictors of recurrence are helpful for patient selection and decision-making on postoperative treatment.

The tumor immune microenvironment (TIME) has been suggested to play an important role in the fight against cancer cells (8). A higher density of tumor-infiltrating $\mathrm{T}$ cells (TILs) has been shown to provide better prognosis and higher sensitivity to chemotherapy for CRC patients $(9,10)$. Although not all types of TILs are prognostic, CD8+ TILs, cytotoxic lymphocytes that can directly kill cancer cells, have been found to correlate with improved survival across a variety of cancers, including localized and metastatic CRC (11). However, the TIME has both pro- and antitumor components in its environment, which means the density of CD8+ TILs alone does not stand for the whole immune landscape.

One of the most notable antagonistic markers is the programmed cell death-ligand 1 (PD-L1). PD-L1 is a protein expressed by a variety of cells in the tumor matrix, and when binding to its receptor the programmed cell death 1 (PD-1) on activated CD8+ TILs, it can down-regulate $\mathrm{T}$ cell activity, leading to immune exhaustion $(12,13)$. Overexpression of stromal PD-L1 has been suggested to be an adverse prognostic factor in many cancers including breast cancer, gastric cancer, and lung cancer, but it has not been validated in CRCLM (14-16). Question arises, therefore, as to whether the combination of CD8+ TIL and PD-L1 expression in the CRCLM is prognostic for patients with CRCLM.

However, determining the prognostic value of this combination in CRCLM remains challenging because there are many other factors affecting the outcome of hepatectomy, including the number of liver metastases and whether the surgery achieves R0 resection (6). Here, we sought to evaluate the prognostic value of the combination of CD8+ TIL and PD-L1 expression in the hepatic TIME, with a specific subset of patients with solitary CRCLM (SCLM) who had undergone R0 resection, and who had no history of preoperative chemotherapy. We present the following article in accordance with the STROBE reporting checklist (available at http://dx.doi.org/10.21037/atm-202762a).

\section{Methods}

\section{Patient selection}

Patients undergoing R0 hepatectomy in Sun Yat-sen University Cancer Center from Jan 2000 to Dec 2016 were reviewed. Primary tumors, if present, were also R0 resected concurrently. Patients with extrahepatic metastases or with a history of systemic chemotherapy within 3 months before liver surgery were excluded. Data on clinicopathological characteristics were extracted from hospital records, including age, sex, preoperative serum carcinoembryonic antigen (CEA), primary tumor location, and the size of liver metastasis. The study was conducted in accordance with the Declaration of Helsinki (as revised in 2013) and was approved by the institutional review board of Sun Yat-sen University Cancer Center (approval number: GZR2017006), and all patients gave written informed consent.

All of the patients were evaluated for the resectability of liver metastases by a multidisciplinary team. R0 resection was defined by negative pathological margins. Intraoperative ultrasound was routinely used to delimit the tumor and to exclude the possibility of covert metastasis. After surgery, adjuvant chemotherapy with oxaliplatin-based regimens was routinely recommended. Follow-up with abdominal computed tomography scan was recommended every 3-6 months within 2 years, and every 6-12 months thereafter.

\section{Immunofluorescent and Immunobistochemical staining}

We used tyramide signal amplification (TSA) multiplexing technique for the quantification of CD8+ T cells. The protocol has been previously described (17). In brief, paraffin-embedded tissues of the resected liver tumors were sliced into 4- $\mu$-thick sections and immunostained with primary antibodies against CD3 and CD8. Nuclei were counterstained with DAPI. The filter cubes used for multispectral imaging were DAPE (440-680 nm), CD3 
Table 1 Baseline characteristics $(n=94)$

\begin{tabular}{|c|c|c|}
\hline Characteristics & $\mathrm{N}$ & Percent \\
\hline \multicolumn{3}{|l|}{ Age (years) } \\
\hline$<60$ & 53 & 56.4 \\
\hline$\geq 60$ & 41 & 43.6 \\
\hline \multicolumn{3}{|l|}{ Sex } \\
\hline Female & 39 & 41.5 \\
\hline Male & 55 & 58.5 \\
\hline \multicolumn{3}{|l|}{ Primary site } \\
\hline Left colon & 25 & 26.6 \\
\hline Right colon & 36 & 38.3 \\
\hline Rectum & 33 & 35.1 \\
\hline \multicolumn{3}{|l|}{ N stage* } \\
\hline Negative & 31 & 37.3 \\
\hline Positive & 52 & 62.7 \\
\hline \multicolumn{3}{|c|}{ Time of metastasis } \\
\hline Metachronous & 40 & 42.6 \\
\hline Synchronous & 54 & 57.4 \\
\hline \multicolumn{3}{|c|}{ Size of metastasis (cm) } \\
\hline$\leq 3$ & 70 & 74.5 \\
\hline$>3$ & 24 & 25.5 \\
\hline \multicolumn{3}{|l|}{ Preoperative CEA } \\
\hline$\leq 5$ & 33 & 37.1 \\
\hline$>5$ & 56 & 62.9 \\
\hline \multicolumn{3}{|c|}{ Postoperative chemotherapy } \\
\hline No & 33 & 35.1 \\
\hline Yes & 61 & 64.9 \\
\hline
\end{tabular}

*, available for 83 patients. CEA, carcinoembryonic antigen.

(520 nm), and CD8 (690 nm). Cells positive for CD3 and CD8 in the tumor were considered CD8+ T lymphocytes, and were quantified as total cell number $\mathrm{X}$ positivity using at least five representative fields at 200X magnification of the tumor areas.

PD-L1 expression was assessed by immunohistochemical staining of these slides using anti-PD-L1 rabbit monoclonal antibody (1:300 dilution, GB11339; Servicebio, Wuhan, China), and was scored by a semiquantitative method, according to the percentage and intensity of positively stained cells (18). The percentage of positively stained cells was scored as: $0,<5 \% ; 1,5-24 \% ; 2,25-49 \% ; 3,50-74 \%$; $4,75-100 \%$, and the intensity staining was scored as: 0 , negative; 1 , weak; 2 , moderate; 3 , strong. The IHC score of PD-L1 expression was the product of two scores.

\section{Patient classifications}

The CD8+ TIL density and PD-L1 expression was each divided into either high or low, according to their respective median value. Since a high CD8+ TIL density indicates strong immune infiltration and low PD-L1 expression suggests less immune exhaustion, we combined the two factors (referred to as CD8-PDL1) and divided the patients into three subgroups: 'Strong' for those with high CD8+ TIL and low PD-L1, 'Weak' for those with low CD8+ TIL and high PD-L1, and 'Mild' for the rest (High CD8+ TIL and High PD-L1 or Low CD8+ TIL and Low PDL1). We then compared their differences in recurrence-free survival (RFS), overall survival (OS), and the performance in predicting recurrence after hepatectomy.

\section{Statistics}

RFS was defined as time from hepatectomy to recurrence or death from any cause, whichever occurred first, and OS was defined as time from hepatectomy to death from any cause. Associations between categorical variables were assessed by Chi-square test, Fisher's exact test, or Wilcoxon rank-sum test, as appropriate. Kaplan-Meier methods were used to estimate RFS and OS probability, and logranks test was used to compare the difference between subgroups. The performance of prediction for RFS was compared using area under the curve (AUC) from receiver operating characteristic (ROC) and time-dependent ROC. All $\mathrm{P}$ values were two-sided, and $\mathrm{P}<0.05$ was considered statistically significant. All analyses were performed in $\mathrm{R}$ (version 3.5.3). Analysis was performed based on available data.

\section{Results}

\section{Baseline characteristics}

Baseline clinicopathological features are listed in Table 1. In total, 94 patients with SCLM that underwent R0 resection were included. Of them, 39 (41.5\%) were female, and the median age was 58 years. Metachronous metastasis was observed in $40(42.6 \%)$ patients. The median size 

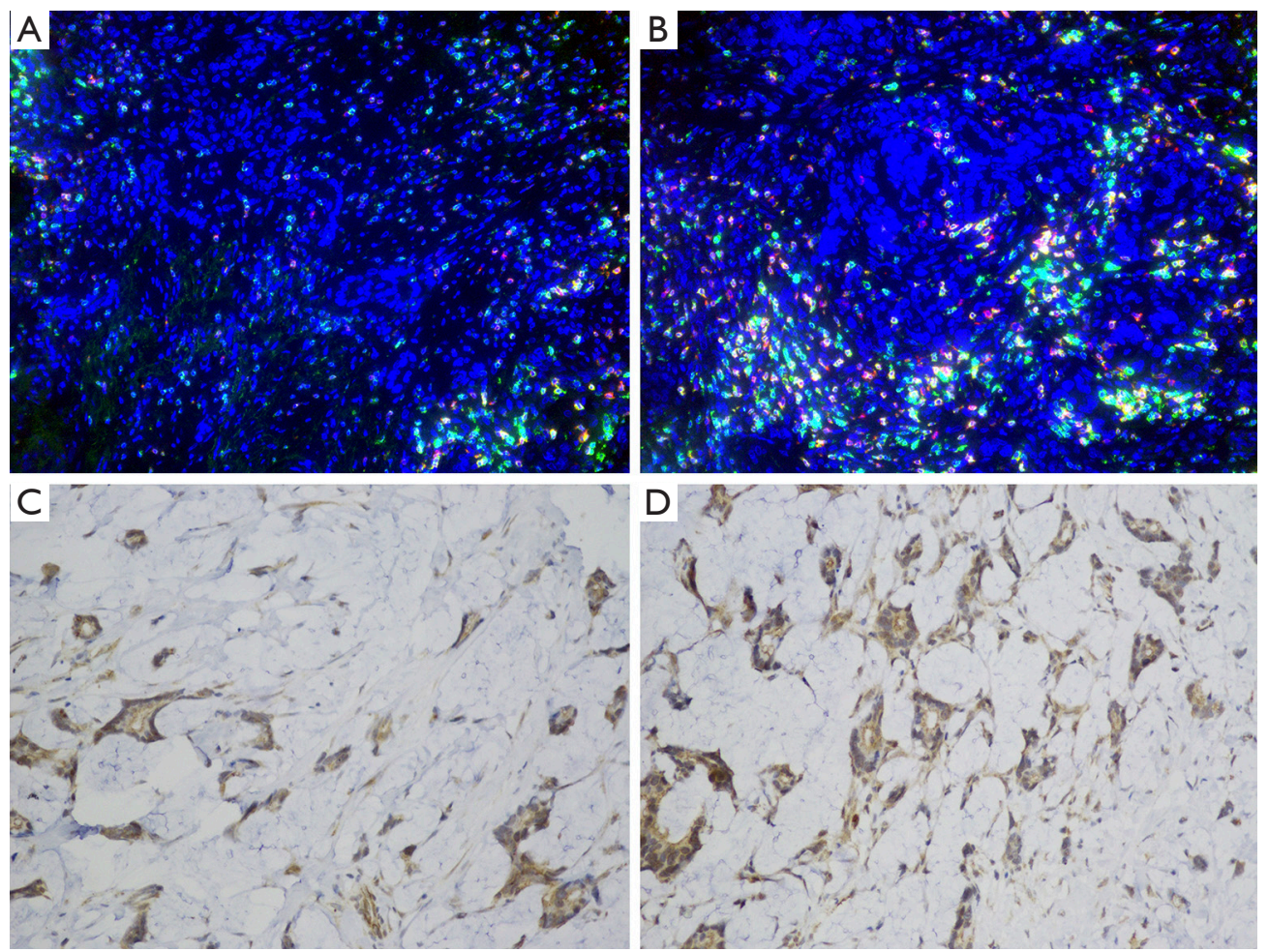

Figure 1 Immunostaining of CD8+ TIL (A, low, 20x; B, high, 20x) and PD-L1 expression (C, low, 40x; D, high, 40x). TIL, tumor infiltrating lymphocytes.

of metastases was $2.0 \mathrm{~cm}$ in diameter, and $24(25.5 \%)$ had metastases $>3 \mathrm{~cm}$. Postoperative chemotherapy was delivered to $61(64.9 \%)$ patients.

\section{Clinicopathological features among CD8, PD-L1, and CD8-PD-L1 subgroups}

Figure 1 shows the low and high density of CD8+ TIL (Figure $1 A, B)$ and PD-L1 expression (Figure1C,D). A low and high CD8+ TIL was observed in 51 (54.3\%) and 43 (45.7\%) patients, respectively. The low and high PD-L1 subgroups each accounted for $50 \%$ of the cases. When CD8+TIL and PD-L1+ cells were combined, the Strong, Mild, and Weak subgroups of CD8-PDL1 were observed in 24 (25.5\%), 42 (44.7\%) and $28(29.8 \%)$ patients, respectively.

Table 2 shows the comparisons of clinicopathological features between the CD8+ TIL and PD-L1 subgroups. High PD-L1 expression was more common among the patients with synchronous metastases $(68.1 \%$ vs. $31.9 \%$, $\mathrm{P}=0.037)$. Similar trend was observed for high CD8+ TIL (74.4\% vs. $25.6 \%, \mathrm{P}=0.002$ ). There were no differences in terms of sex, primary site, lymph nodes involvement, preoperative serum CEA level, and the size of metastasis. For the three subgroups of CD8-PDL1, all of the above factors were well balanced.

\section{Comparisons of survival and predictive performance}

After a median follow up of 36.4 (1.6-143.3) months, recurrence and death occurred in 38 and 25 patients, respectively. The median RFS was 72.3 months, and the median OS was not reached (Table 3). Of the 38 recurrences, $22(57.8 \%)$ were confined in the liver; the other $16(42.2 \%)$ involved extrahepatic sites.

In the subgroup analysis (Figure 2), patients with a high CD8+ TIL had significantly longer RFS than patients with a low CD8+ TIL (median: not reached vs. 55.8 months, 3 -year RFS $71.6 \%$ vs. $55.3 \%, \mathrm{P}=0.018$ ). In contrast, patients with low PD-L1 expression had longer RFS than patients with high PD-L1 expression (median: not reached $v s$. 24.9 months, 3 -year RFS $88.6 \%$ vs. $38.4 \%, \mathrm{P}<0.001$ ). Patients with low PD-L1 expression had significant longer OS than patients with high PD-L1 expression, with the 3 -year OS of $92.9 \%$ vs. $65 \%(\mathrm{P}<0.001)$. 
Table 2 Comparison of baseline features between CD8 and PD-L1 subgroups

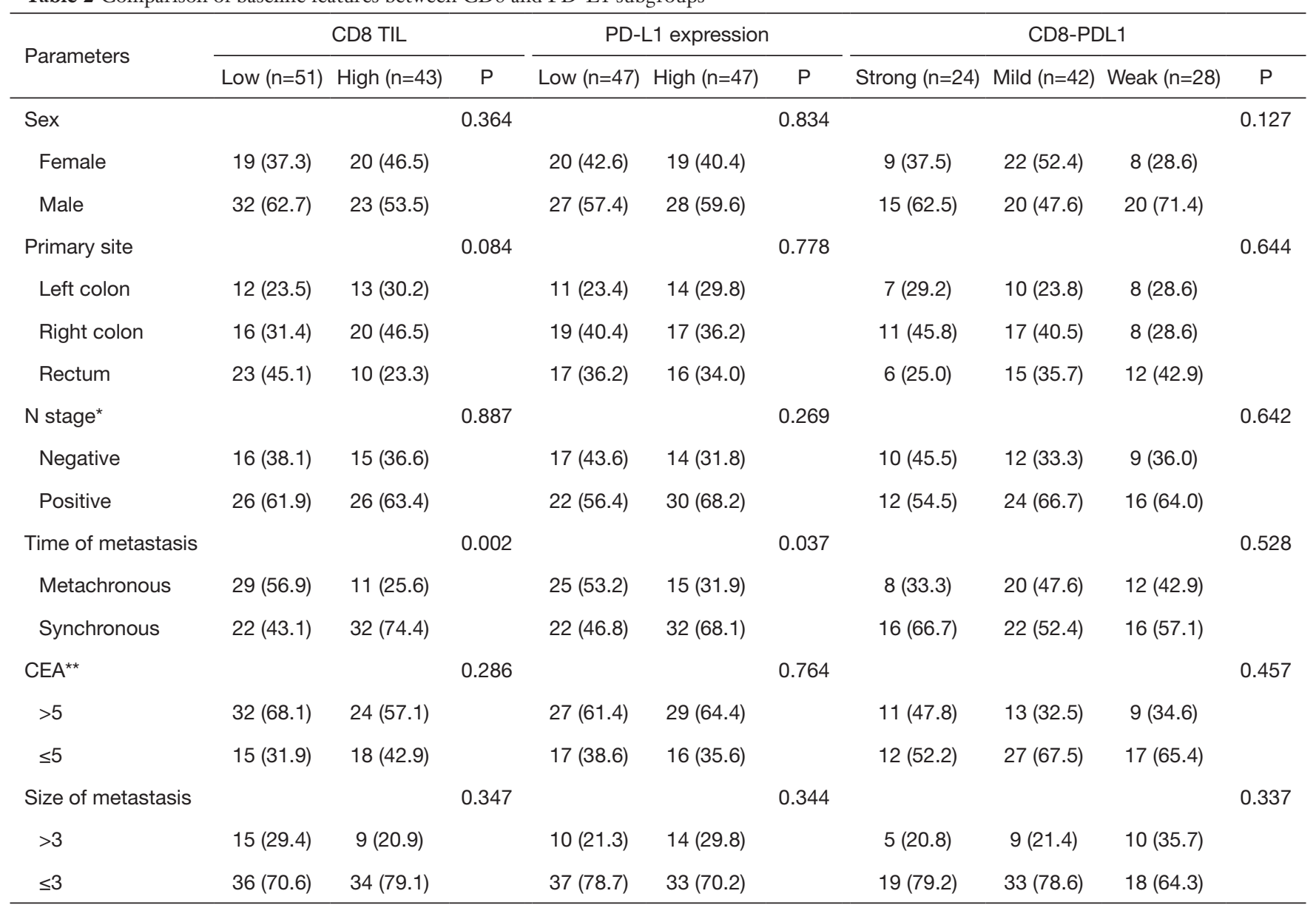

*, available for 83 patients; ${ }^{*}$, available for 89 patients. CEA, carcinoembryonic antigen; TIL, tumor infiltrating lymphocytes; CD8-PDL1, combination of CD8 TIL and PD-L1 expression.

For the CD8-PDL1, median RFS and OS were not reached for the Strong and Mild subgroups, and were 13.6 and 43.2 months respectively for the Weak group $(\mathrm{P}<0.001)$. The 3-year RFS rate in the Strong group was significantly higher than that in the Mild and Weak groups $(89.5 \%$ vs. $71.7 \%$ and $28.8 \%, \mathrm{P}<0.001)$, as was the 3 -year rate of OS (93.8\% vs. $81.8 \%$ and $61.6 \%, \mathrm{P}<0.001$ ) (Figure 2 ). The preoperative CEA level and postoperative chemotherapy history were not associated with RFS and OS.

In the ROC analysis (Figure $3 A$ ), the CD8-PDL1 displayed larger AUC than the CD8 and PD-L1 alone [0.79 (0.711-0.879) vs. $0.64(0.543-0.739)$ and $0.77(0.679-$ $0.851)]$, suggesting greater ability to predict recurrence after $\mathrm{R} 0$ resection for CRCLM. In the time-dependent ROC analysis, the CD8-PDL1 showed good predictive performance at multiple time points $(12,24,36,48,60$, and 72 months), and the AUC stabilized after 60 months (Figure 3B).

\section{Discussion}

The role of TIME in carcinogenesis is complex because there are both pro- and anti-tumor immunological components in the same environment (19). In this study, we confirmed that the density of CD8+ TIL was a favorable prognostic factor of RFS and OS for patients with SCLM undergoing R0 resection. Moreover, when it was combined with PD-L1 expression, the factor CD8-PDL1 was able to discriminate patients with different outcomes, and its predictive accuracy for RFS was superior to that of CD8+ TIL alone.

Although recurrence is common among patients with 
Table 3 Survival analysis of RFS and OS

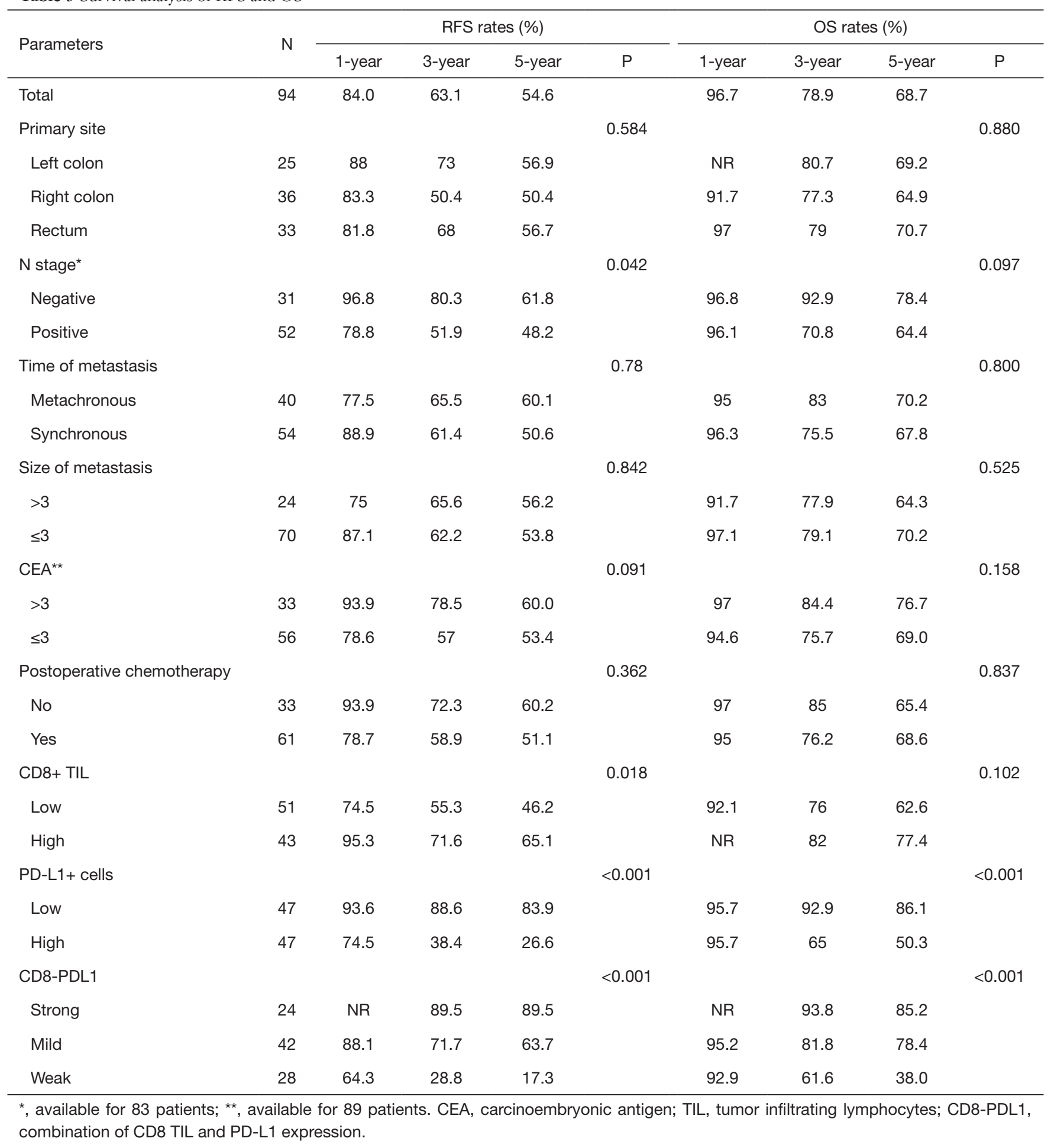



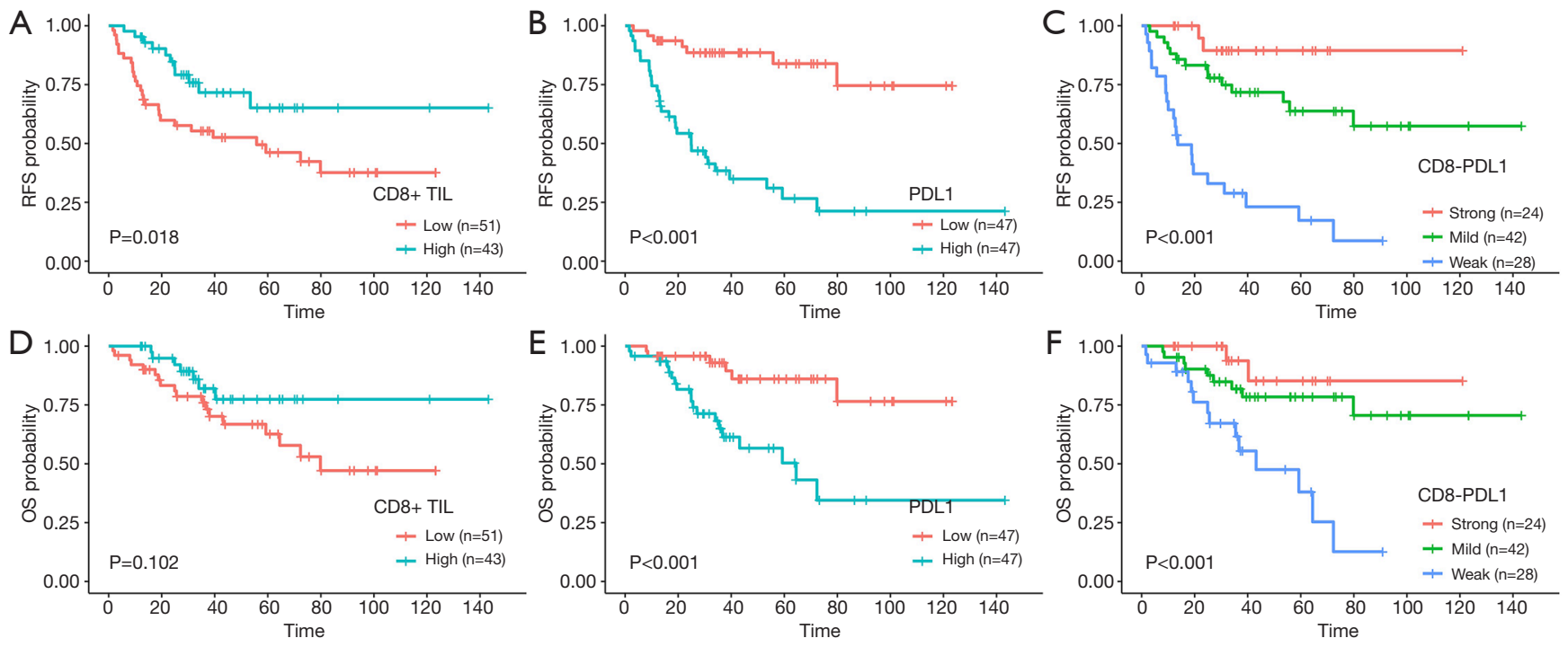

Figure 2 RFS and OS for patients with different CD8+ TIL (A and D), PD-L1 expression (B and E), and CD8-PDL1 (C and F). RFS, recurrence-free survival; OS, overall survival; TIL, tumor infiltrating lymphocytes; CD8-PDL1, combination of CD8+ TIL and PD-L1 expression.
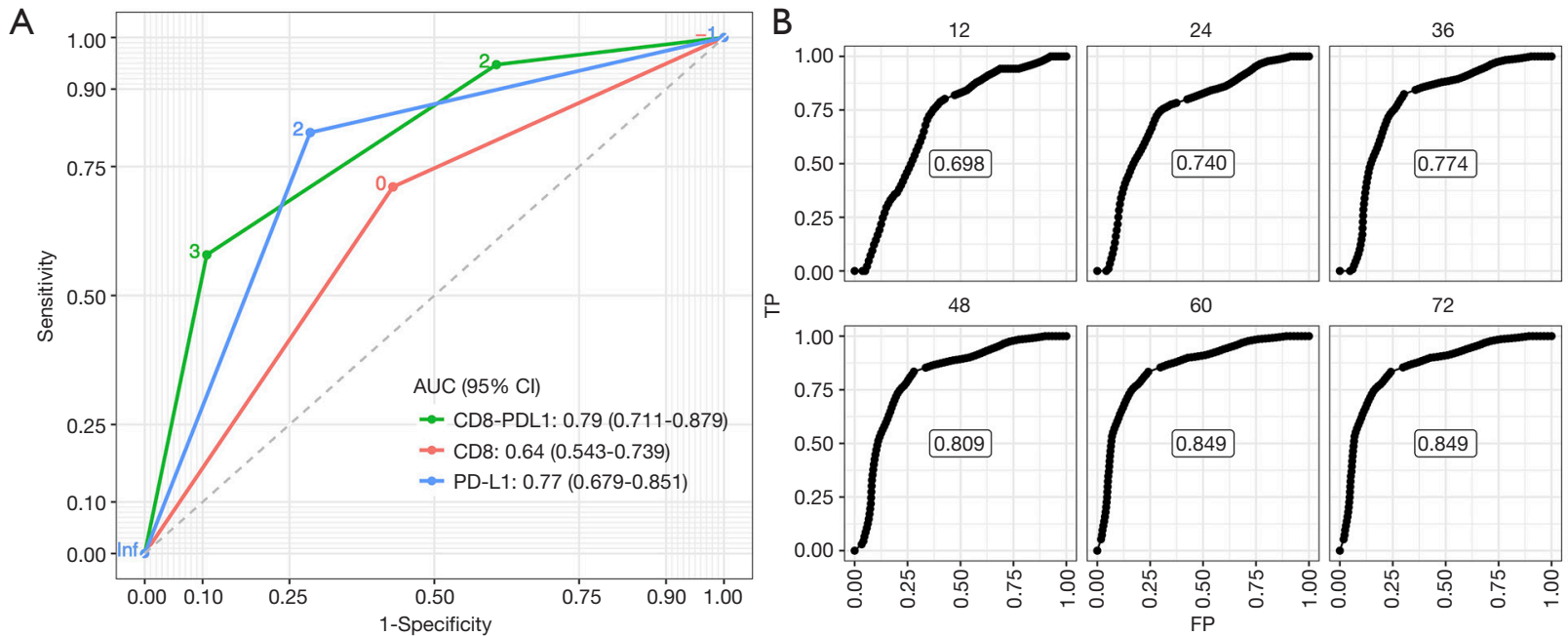

Figure 3 Prediction performance of CD8, PD-L1, and CD8-PDL1. (A) ROC of CD8+ TIL, PD-L1 expression and CD8-PDL1 for predicting RFS. (B) Time-dependent ROC for CD8-PDL1 for predicting RFS at 12, 24, 36, 48, 60, and 72 months after hepatectomy. ROC, receiver operating curve; TIL, tumor infiltrating lymphocytes; CD8-PDL1, combination of CD8+ TIL and PD-L1 expression.

CRCLM undergoing liver resection, those with solitary metastasis have longer survival. Fong et al. found that patients with solitary CRCLM had a 5-year OS rate of 44\% after liver surgery, significantly higher than that of patients with $\geq 2$ CRCLM (6). In our study, all patients had solitary CRCLM, and the 5 -year OS rate were $68.7 \%$, higher than previously reported. The major reason for this may be that the sizes of the liver metastases in this study were relatively small, with a median diameter of $2.0 \mathrm{~cm}$ and only one fourth being $\geq 3 \mathrm{~cm}$. According to previous studies, patients with CRCLM $<3 \mathrm{~cm}$ have longer survival than those with CRCLM $\geq 3 \mathrm{~cm}(20)$.

The density of CD $8+$ TIL, in both primary and metastatic lesions, have been proven to be a prognostic 
factor of survival in a variety of cancers (11). In CRC, Pagès et al. found that a higher density of CD8+ TIL in primary CRC was associated with fewer signs of early metastatic invasion and better survival, and Mlecnik et al. found that the predicting performance of TIL was better than that of MMR status and TNM stage (21-23). Consistent with those reports, our results showed that patients with a higher CD8+ TIL had a better outcome, with 3-year RFS rate being $71.6 \%$ vs. $55.3 \%$. Moreover, since TIL varies between liver metastases and can be affected by chemotherapy, by incorporating only patients with solitary metastasis and with no history of neoadjuvant chemotherapy, our study provided unequivocal high-quality evidence supporting the favorable role of TIL in CRCLM $(24,25)$.

However, the density of TIL is not the only factor that determines the immune activity in the TIME; there are other factors affecting the function of immune cells, among which PD-L1 expression is the most known one. The interaction between PD-1 and PD-L1 puts a brake on the activated CD8+ T cells, inhibiting their release of cytotoxic cytokines such as Granzyme B and Interferon- $\gamma$ (12). In human hepatocellular carcinoma, Gao et al. demonstrated that overexpression of PD-L1 was significantly associated with tumor aggressiveness and a high recurrence rate after resection (26). In gastric cancer, Thompson et al. found that, tumors with an increased CD8+ TIL were usually accompanied by overexpression of PD-L1, and that PDL1 expression counteracted the immunological effect of TIL, leading to worse survival in patients with a higher density of TIL (14). Other studies have found that tumorassociated macrophages and several cytokines are linked to immune cell exhaustion in the tumor microenvironment $(27,28)$. Together, these findings suggest that the immune environment as a whole, rather than the aggregation of $\mathrm{T}$ cells alone, is responsible for the anti-cancer effect of the immune system.

In this study, when classified into two groups according to PD-L1 expression, patients with a higher PD-L1 had significantly worse RFS and OS than those with a lower PD-L1, supporting the immunosuppressive role of PDL1 in the setting of CRCLM. Moreover, when CD8+ TIL and PD-L1 expression were combined, CD8-PDL1 was able to divide patients into three groups with different outcomes: those with the strong immune reaction (high CD8+ TIL and low PD-L1) had the longest survival, while those with the mild immune reaction (high CD8+ TIL and high PD-L1 or low CD8+ TIL and low PD-L1) had shorter survival-but it was still longer than those with the weak immune reaction (low CD8+ TIL and high PDL1). In addition, with regard to the predicting accuracies for recurrence, the model of CD8-PDL1 had significantly larger AUCs than the CD8 model. In time-dependent ROC analysis, CD8-PDL1 also showed high accuracy for predicting recurrence. Therefore, CD8-PDL1 can be used as a prognostic marker for patients with SCLM undergoing hepatectomy, thus providing guidance for their postoperative treatment.

Our study has some limitations. First, it is a single-center study and its sample size is relatively small, therefore the results it yielded should be validated in large-scale cohorts. Second, apart from CD8+ TIL and PD-L1 expression, there are many other factors that can influence immune reaction, including the infiltration of macrophages, neutrophils and other cells (11). These were not taken into account in the study. Last, the outcome of metastatic CRC is also affected by the genotype of some genes such as KRAS and BRAF (29). These two genes were tested in less than one fifth of the patients and thus were not analyzed.

\section{Conclusions}

The density of CD8+ TIL and PD-L1 expression were predictors of RFS for patients with solitary CRCLM undergoing R0 liver resection. The TIME, as measured by PD-L1 expression and CD8+ TIL density, was able to further discriminate these patients with different outcomes, providing reliable guidance for postoperative treatments.

\section{Acknowledgments}

Funding: The study was funded by National Natural Science Foundation of China (No. 81772595), Natural Science Foundation of Guangdong Province, China (No. 2018A030310260), Natural Science Foundation of Guangdong Province (grant No. 2017A030310204), and Bethune-Ethicon Excellence Surgery Fund Project (No. HZB-20181119-27). The authenticity of this article has been validated by uploading the key raw data onto the Research Data Deposit public platform (www. researchdata.org.cn), with the approval RDD number as RDDA2020001606.

\section{Footnote}

Reporting Checklist: The authors have completed the STROBE reporting checklist. Available at http://dx.doi. 
org/10.21037/atm-20-2762a

Data Sharing Statement: Available at http://dx.doi. org/10.21037/atm-20-2762a

Peer Review File: Available at http://dx.doi.org/10.21037/ atm-20-2762a

Conflicts of Interest: All authors have completed the ICMJE uniform disclosure form (available at http://dx.doi. org/10.21037/atm-20-2762a). All authors report grants from National Natural Science Foundation of China, grants from Natural Science Foundation of Guangdong Province, China, during the conduct of the study.

Ethical Statement: The authors are accountable for all aspects of the work in ensuring that questions related to the accuracy or integrity of any part of the work are appropriately investigated and resolved. The study was conducted in accordance with the Declaration of Helsinki (as revised in 2013) and was approved by the institutional review board of Sun Yat-sen University Cancer Center (approval number: GZR2017-006). All written informed consent was obtained.

Open Access Statement: This is an Open Access article distributed in accordance with the Creative Commons Attribution-NonCommercial-NoDerivs 4.0 International License (CC BY-NC-ND 4.0), which permits the noncommercial replication and distribution of the article with the strict proviso that no changes or edits are made and the original work is properly cited (including links to both the formal publication through the relevant DOI and the license). See: https://creativecommons.org/licenses/by-nc-nd/4.0/.

\section{References}

1. Bray F, Ferlay J, Soerjomataram I, et al. Global cancer statistics 2018: GLOBOCAN estimates of incidence and mortality worldwide for 36 cancers in 185 countries: Global Cancer Statistics 2018. CA Cancer J Clin 2018;68:394-424.

2. Chen $W$, Zheng R, Baade PD, et al. Cancer statistics in China, 2015: Cancer Statistics in China, 2015. CA Cancer J Clin 2016;66:115-32.

3. Malvezzi M, Carioli G, Bertuccio P, et al. European cancer mortality predictions for the year 2018 with focus on colorectal cancer. Ann Oncol 2018;29:1016-22.
4. Hughes KS, Simon R, Songhorabodi S, et al. Resection of the liver for colorectal carcinoma metastases: A multiinstitutional study of patterns of recurrence. Surgery 1986;100:278-84.

5. Steele G, Ravikumar TS. Resection of hepatic metastases from colorectal cancer. Biologic perspective. Ann Surg 1989;210:127-38.

6. Fong Y, Fortner J, Sun RL, et al. Clinical score for predicting recurrence after hepatic resection for metastatic colorectal cancer: analysis of 1001 consecutive cases. Ann Surg 1999;230:309-18; discussion 318-21.

7. Van Cutsem E, Cervantes A, Adam R, et al. ESMO consensus guidelines for the management of patients with metastatic colorectal cancer. Ann Oncol 2016;27:1386-422.

8. Binnewies M, Roberts EW, Kersten K, et al. Understanding the tumor immune microenvironment (TIME) for effective therapy. Nat Med 2018;24:541.

9. Galon J, Costes A, Sanchez-Cabo F, et al. Type, density, and location of immune cells within human colorectal tumors predict clinical outcome. Science 2006;313:1960-4.

10. Fridman WH, Galon J, Pages F, et al. Prognostic and Predictive Impact of Intra- and Peritumoral Immune Infiltrates. Cancer Res 2011;71:5601-5.

11. Gooden MJM, de Bock GH, Leffers N, et al. The prognostic influence of tumour-infiltrating lymphocytes in cancer: A systematic review with meta-analysis. Br J Cancer 2011;105:93-103.

12. Keir ME, Butte MJ, Freeman GJ, et al. PD-1 and Its Ligands in Tolerance and Immunity. Annu Rev Immunol 2008;26:677-704.

13. Freeman GJ, Long AJ, Iwai Y, et al. Engagement of the Pd-1 Immunoinhibitory Receptor by a Novel B7 Family Member Leads to Negative Regulation of Lymphocyte Activation. J Exp Med 2000;192:1027-34.

14. Thompson ED, Zahurak M, Murphy A, et al. Patterns of PD-L1 expression and CD8 T cell infiltration in gastric adenocarcinomas and associated immune stroma. Gut 2017;66:794-801.

15. Mu CY, Huang JA, Chen Y, et al. High expression of PD-L1 in lung cancer may contribute to poor prognosis and tumor cells immune escape through suppressing tumor infiltrating dendritic cells maturation. Med Oncol 2011;28:682-8.

16. Muenst S, Schaerli AR, Gao F, et al. Expression of programmed death ligand 1 (PD-L1) is associated with poor prognosis in human breast cancer. Breast Cancer Res Treat 2014;146:15-24.

17. Peng J, Wang Y, Zhang R, et al. Immune Cell Infiltration 


\section{Page 10 of 10}

in the Microenvironment of Liver Oligometastasis from Colorectal Cancer: Intratumoural CD8/CD3 Ratio Is a Valuable Prognostic Index for Patients Undergoing Liver Metastasectomy. Cancers 2019;11:1922.

18. Taylor CR, Levenson RM. Quantification of immunohistochemistry? Issues concerning methods, utility and semiquantitative assessment II. Histopathology 2006;49:411-24.

19. Joyce JA, Fearon DT. T cell exclusion, immune privilege, and the tumor microenvironment. Science 2015;348:74-80.

20. Leung U, Gönen M, Allen PJ, et al. Colorectal Cancer Liver Metastases and Concurrent Extrahepatic Disease Treated With Resection. Ann Surg 2017;265:158-65.

21. Pagès F, Berger A, Camus $M$, et al. Effector memory $T$ cells, early metastasis, and survival in colorectal cancer. $\mathrm{N}$ Engl J Med 2005;353:2654-66.

22. Mlecnik B, Tosolini M, Kirilovsky A, et al. Histopathologic-based prognostic factors of colorectal cancers are associated with the state of the local immune reaction. J Clin Oncol 2011;29:610-8.

23. Mlecnik B, Bindea G, Angell HK, et al. Integrative Analyses of Colorectal Cancer Show Immunoscore Is a Stronger Predictor of Patient Survival Than Microsatellite
Xiao et al. PD-L1 expression and CD8 TIL predict RFS for CRCLM

Instability. Immunity 2016;44:698-711.

24. Halama N, Spille A, Lerchl T, et al. Hepatic metastases of colorectal cancer are rather homogeneous but differ from primary lesions in terms of immune cell infiltration. Oncoimmunology 2013;2:e24116.

25. Mlecnik B, Van den Eynde M, Bindea G, et al. Comprehensive Intrametastatic Immune Quantification and Major Impact of Immunoscore on Survival. J Natl Cancer Inst 2018;110:97-108.

26. Gao Q, Wang XY, Qiu SJ, et al. Overexpression of PDL1 significantly associates with tumor aggressiveness and postoperative recurrence in human hepatocellular carcinoma. Clin Cancer Res 2009;15:971-9.

27. Halama N, Zoernig I, Berthel A, et al. Tumoral Immune Cell Exploitation in Colorectal Cancer Metastases Can Be Targeted Effectively by Anti-CCR5 Therapy in Cancer Patients. Cancer Cell 2016;29:587-601.

28. Aras S, Zaidi MR. TAMeless traitors: Macrophages in cancer progression and metastasis. Br J Cancer 2017;117:1583-91.

29. Dekker E, Tanis PJ, Vleugels JLA, et al. Colorectal cancer. Lancet 2019;394:1467-80.

Cite this article as: Xiao B, Peng J, Wang Y, Deng Y, Ou Q, Wu X, Lin J, Pan Z, Zhang L. Prognostic value of tumor infiltrating lymphocytes combined with PD-L1 expression for patients with solitary colorectal cancer liver metastasis. Ann Transl Med 2020;8(19):1221. doi: 10.21037/atm-20-2762a 